\title{
Educational XRealities
}

\author{
Martijn Stellingwerff ${ }^{1}$ \\ ${ }^{1}$ Form \& Modelling Studies, Faculty of Architecture, TU-Delft, the Netherlands
}

\begin{abstract}
XRealities is a recently approved pedagogic fellowship project that will run for two years, during 2019 and 2020. This paper will introduce the project plan, some early experiments and expectations. XRealities relates to the many real and digital situations that we encounter in our digitally expanded life experiences. In contemporary education, we notice a problematic situation with abundant screens. People sit next to each other and stare at screens, looking for contact and content. We describe the current dichotomy between latent online learning materials and potential real-world experiences in campus education. The XRealities project is focused on finding alternative approaches for educational settings in which both realworld social interactions and virtual content provide new forms of intertwined learning experiences. We assume there is huge educational potential in the right juxtaposition of visual digital and real-world experiences and the embodied interaction within this augmented reality.
\end{abstract}

\section{Introduction - screens in education}

The essence of education is bringing the world to the student. Educators assist in the exploration of world-aspects and challenge the learner to find and train the right responses to that world. In education, we make use of collections, descriptions, recordings, images, schemes, models, simulations and prototypes to represent selections of the world in the best possible ways, although real experiences, such as excursions, practical work and creative production of full-scale objects also belong to the educational curriculum, screens, in different sizes, take an increasingly dominant role in classes, lecture rooms, design studios and even in workshops.

With this French year of the Architectural Envisioning conference in mind, we could refer to the famous Lascaux Cave as an ultimate educational representation. The beautiful ancient cave drawings could be considered an early screen that depicts hunting strategies and characteristics of the most impressive animals in the field. This 360-degree cave experience is still as impressive as contemporary screens, with for example a 360-degree video about "Swimming with Giants" [1]. Huge buffalos and majestic deer, impressive whales and gigantic squids ... they all have to compete with a massive amount of cute cat pictures on mobile phones.

Between the cave drawings and a spherical video experience on YouTube, we can consider a whole range of innovations in screen technology. Moses, for example, used mobile stone tablets to teach biblical laws. Flintstone was a thinner update that was also supporting 
the use of chalk pencils, which brought us 'easy-undo' features. The modern blackboard can still be found in school classes. A parallel technology focused on very thin foldable and bendable material, it can still be found and is known as ... paper. Paper works with a real ink-based stylus, also known as pen. Eventually, with the efforts of clerks, human computers, and monks, a lot of knowledge was pencilled on paper and stored in libraries (physical repositories, comparable to cloud server buildings). The invention of printing technologies helped spreading our knowledge. The rest is prehistoric.

In contemporary education, we find screens in abundance. The ubiquitous screens are our predominant digital windows to the world. Four types of screens can be distinguished: screens with beamers and TV-sets for group presentations, laptop screens for sitting people, and mobile device screens to fill in all the time we cannot use the first two types of screen. VR goggles can be seen as a form of ultimate screen, close to the eyes, for full immersion.

\section{Problem statement}

Screens dominate and distract from valuable learning experiences involving collaboration, prototypes, simulations, tests and topical conversations. Contemporary physical and digital learning environments exist side by side but lack integration.

The lack of integrated physical and digital learning environments becomes apparent in social aspects, education spaces, learning materials and the deliverables (case studies, designs, proposals, models, prototypes, etc.):

- In the social behaviour of teachers and students, we can see the constant demand for attentiveness between real-near and virtual-remote collaborators. Screens and headphones isolate individuals from a group. Phone-screens often interrupt a good conversation. At the same time, there are global social ties that can come about, completely independent of place and time.

- Physical and digital education spaces offer totally different experiences. Lecture rooms, classrooms and studios bring uniquely focused events while online learning platforms (e.g. Blackboard, Brightspace and edX) support information exchange in a durable and accessible way.

- A third lack of integration can be found in educational collections, such as physical repositories for publications, samples, exhibition objects, tools and their digital counter parts with collections of open textbooks, images, 3D objects, code and all sorts of digital assets. Online learners and campus learners have different experiences.

- The lack of integration is also apparent between, on the one hand, physical objects in education laboratories and workshops and, on the other hand, virtual prototypes, digital performance testing, simulation, AI and VR.

The dichotomy of the physical and digital environments is already somewhat solved by deliberate disconnecting them in time and place, for example by introducing Blended Learning. The idea of blended learning is that the student prepares at home, using online learning materials, while contact hours are used more effectively for direct conversations, collaborations and exercises with peers and tutors. It should be noted that the word blended in blended learning indicates a combination of two more or less separate modes of learning, while the XRealities project aims to truly intertwine the experience of context and peers with digital content within a digitally augmented environment.

Other approaches partially integrate digital interactions and integration via screen sharing, e.g. using Clickshare [2], and classroom voting systems, e.g. Mentimeter [3], Kahoot 
[4] and Feedback Fruits [5]. A good tool that truly integrates group collaborations with interactive screens is e.g. Nureva [6]. By combining physical actions of participants, using huge touch screens, Nureva brings the notion of shared idea creation to a visually consistent group experience.

\section{The Educational XRealities project}

Despite aforementioned good exceptions, we notice a huge mismatch between the abundant screens, online content and the real-world aspects of active, contextual, socially situated education. One new and ultimate type of see-through screen technology could solve this dichotomy between the real world and the digital world: Augmented Reality (AR). Although AR consists of screen technologies, the screens more or less disappear and give way to shared, digitally augmented, spatial experiences.

At the moment, we see rapid changes in the ways students use data from 'the cloud' and in the ways they form alternative networks for meeting and assisting each other. Soon, the changes will bring 'goggles and googles' (devices and data clouds), enhanced by artificial intelligence, that will have unprecedented impact on many details in our life. The setting of our education will change drastically.

In education, we have individuals and communities of people with different roles. They meet online and in social spaces such as classrooms, labs and studios. They make use of real and digital learning materials, including tools, instruments, data sets, models, prototypes, algorithms and simulations. Each gathering of teaching and learning people, in a composition of virtual or physical spaces, with a specific choice of learning materials, can be called an 'educational setting'. In the XRealities project, we will describe educational settings that allow us to investigate a range of future scenarios.

Different forms of Augmented Reality (AR) can be used to superimpose physical environments and digital content, to integrate prototypes and human actions. The AR environments create new educational settings that will be valued relative to existing solutions.

$\mathrm{XR}$ is a collective term for a range of key enabling technologies that will soon change the realm of education in exciting and challenging ways. XR encompasses VR and AR, but also the 'Real World' and the world of our imagination. The terms AR, VR and XR are currently adopted in different ways and the definitions vary. Sometimes XR is being disputed and disliked, while recently the term 'spatial computing' becomes more popular.

In our project, XR stands for XRealities. This will be a number of eXperimental education settings that range from digitally augmented university campuses to full-immersive VR settings that allow collaborations between participants from all over the world. Although technology is an enabling factor in the experiments, the outcomes will especially regard pedagogic insights for the organization of future campus- and online education.

\subsection{Project plan}

During the first year, we would like to make an ongoing and expanding augmented campus exhibition. This would start with examples of persistent AR content within the Teaching Lab. Based on those examples, other teachers would be invited and supported to build their own topical campus exhibition and eventually their own AR learning materials. At a certain 
moment, the campus will be full of exciting examples in line with the Open Science and Open Education policy $[7,8]$ of our university. Partial exhibitions could be built with students from different faculties or related to events, such as the International Festival of Technology, a conference, or they could play a role during the welcoming of new students at the start of the academic year.

During the second year, we would like to focus on meetings in VR and AR on top of the augmented exhibition content. This would involve making personalized avatars for teachers and students. An online XR conference would complete the project. Such a conference would offer local and global exchange of experiences, without the footprint of travel and without scheduling problems and absence.

The field of XR is developing rapidly. Developer tools are available and specific experiments can be created relatively easily. This implies that there is not a large risk in the actual technical challenge. The experiments depend more on the proper description of educational objectives and the right choice of content and interaction.

\subsection{Experiments}

Experiments are key in the XRealities project. We already started finding potential qualities of AR in relation to simulated education settings within the interior building context of our Teaching Lab. This laboratory opened in September 2017 [9], and it offers a creative meeting and working space for all educators and support staff at the TU Delft. The open, warm yellow interior of the Teaching Lab provides a unique spatial décor for meeting colleagues, and it is also the place for many sorts of training, sharing and experimentation. An additional and unexpected benefit comes from the wood chips in the yellow coated Oriented Strand Board (OSB), which is used all over the place. The subtle but unique OSB pattern in the floor and wall finish turns out to be ideal as a tracking pattern for AR applications.

We will get started by experimenting with an iOS app that allows to directly compose and test AR content. After an in-depth study of the available AR authoring platforms, we did not choose one of the main players, like Unity or Unreal Engine, nor did we choose to dive into programming challenges, using ARkit or ARcore. Instead, we chose to experiment with a new app and platform that allows to directly create and explore interactive AR experiences using mobile devices such as iPhones and iPads. The app 'Torch' [10] allows to quickly place and combine different 3D models and then add interactions and changes in scenes. This frees us from programming efforts and therefore makes it possible to be playful, quick, responsive and iterative in building and evaluating different educational AR prototypes. Torch comes with many improvements in each new app update and their roadmap reassures that many extensions to new and existing platforms and possibilities to export to the aforementioned Unity or Unreal Engine authoring tools will be added.

The experiments will include content from some of the various disciplines in the faculties of our university. We will connect the content to different learning goals, as described and schematised in Bloom's revised taxonomy $[11,12]$. The range of learning objectives will be systematically explored during the experiments. In that way, we will explore how well the objectives in Bloom's taxonomy relate to the medium and user experiences offered by AR.

To illustrate the project, we could already describe one of our first experiments. This will be a small exhibition about changeable furniture. We will digitize chairs and one specific chaise longue. The real pieces of furniture belong to our large faculty collection of design 
chairs. Most of these chairs are designed by architects. The selected chairs will be augmented by $3 \mathrm{D}$ representations that demonstrate the state changes of the chairs. Some chairs can be changed by hinges, others can be decomposed and fit in another way around. The real chairs cannot constantly be touched. Therefore, the AR experiment would be successful if it teaches the different options. Referring to Bloom's learning objectives, the augmented chair exhibition should allow the visitor of the exhibition to analyse the chair positions and apply the understanding by moving or rotating the digital model representation in order to bring it in the alternative state.

\subsection{Expectations of the project}

We expect that Bloom's taxonomy in the 'Cognitive Process Dimension' allows us to come up with AR prototypes that facilitate and support each of Bloom's six learning objectives: remember, understand, apply, analyse, evaluate, create. We expect to be able to implement these learning objectives in a number of case exhibitions. Follow-up research would be needed to further understand the workings and effects of AR as an educational tool or medium. Given the predominant visual nature of most AR experiences, we assume that the successfulness of the learning objectives depends to a large extent on the subject matter of the education. In that regard, we will select cases that relate to design and understanding of complex spatial subjects. The tools within the AR environment should relate to the objectives. For example, if a learner needs to analyse a situation, this brings different prerequisites, compared to if he or she needs to learn to create something.

Within the 'Knowledge Dimension' of Bloom's taxonomy, we expect that conceptual and procedural knowledge relates better to AR than the factual and metacognitive knowledge. Facts can also be learned in less advanced ways, using descriptions, schemes, and illustrations. Metacognitive knowledge combines several aspects, and we assume that this type of knowledge needs more distance and reflection; we assume that will not be available in the direct, active modes of learning that belong to an AR experience.

Although we confront ourselves with a complex taxonomy with many aspects and interpretations, it is expected that this will not harm the richness of findings for this project. We are aware that within the given time, only the surface of this new medium can be explored. The width of scope, open to collaborations, experimentation, twists and turns excites. A new medium with so much potential should be explored in open and playful ways, while the findings should be noted, communicated and exhibited using evocative examples.

\subsection{Deliverables}

The educational fellowship within which this project will be carried out requires dissemination of knowledge and direct application in education. An added request is that the explored techniques should preferably suit the education of large numbers of learners, online and on campus. These requirements can be fulfilled if the expectations raised by the AR industry will also be fulfilled. This notion should be clear, because technical research is not proof of knowledge, instead it is explorative and creative and leads to new insights. The decision to focus on Blooms taxonomy while using available authoring tools is a practical choice with positive opportunistic sense for what might become our new way of teaching.

Therefore, the deliverables are open to surprise and influences from colleagues, students and anyone involved. We aim to make several AR exhibitions within the Teaching Lab and on the streets and squares of the TU-Delft campus. In the process towards the exhibition, we 
will record AR videos to disseminate early findings and we will invite teaching staff to workshops, and we will initiate special sessions to focus on their educational needs. Early results will be shown during the 2019 EAEA conference in Nantes.

Apart from the collaborations and experiments, we expect new insights regarding spatial user interfaces, new insights regarding juxtaposition of context and digital visualisations, and new insights regarding perception and the dynamic perspective within AR.

Finally, we expect new insights based on the creation of a large number of experiments. Those insights will be communicated in a new online course regarding the workflow and best practices for finding, making, using, and sharing 3D assets for education.

\section{References}

1. American Museum of Natural History, Swimming With Giants, published October 20th, 2018, https://youtu.be/wOsq7fQ9Z3A

2. Clickshare, https://www.barco.com/en/clickshare

3. Mentimeter, https://www.mentimeter.com

4. Kahoot, https://kahoot.com

5. Feedback Fruits, https://feedbackfruits.com/home

6. Nureva, https://www.nureva.com

7. Open Science: https://www.tudelft.nl/en/library/current-topics/tu-delft-open-science/

8. Open Education: https://ocw.tudelft.nl

9. Valkenburg, W. van; http://www.e-learn.nl/2017/09/28/opening-tu-delft-teaching-lab

10. Torch, https://www.torch.app/

11. Anderson, Lorin W.; Krathwohl, David R.; Bloom, Benjamin S; 2001, A taxonomy for learning, teaching, and assessing: a revision of Bloom's taxonomy of educational objectives, New York: Longman.

12. Revised Bloom's Taxonomy, http://www.celt.iastate.edu/teaching/effective-teachingpractices/revised-blooms-taxonomy/ 